\title{
On Translating the Poetic Images from Chinese into English
}

\author{
Mingquan Zhang \\ School of Foreign Languages, Jiangsu University \\ Zhenjiang 212013, Jiangsu Province, China \\ Tel: 86-511-8537-9133Ｅ-mail: mingquanzhang68@163.com \\ Weiqiang Mao \\ School of Foreign Languages, Jiangsu University \\ Zhenjiang 212013, Jiangsu Province, China
}

The Research is financed by "Humanities and Social Sciences Key Program of Jiangsu University" (No. JDR2006A11) and "Jiangsu Province Institutions of Higher Learning Social Sciences Program". (No. 06SJD750023)

\begin{abstract}
Despite the concern over the loss of poetic images in the translation process, the translation practice of poetry never ceases. As the result of different approaches to the poetic imagery, the poetic images might be preserved, lost or replaced by others. The hermeneutic approach, with a view to preserving the alterity of an alien culture, would like the original image to be preserved as much as possible. The comparative literature approach is concerned with the misreading unavoidable in the translation process and cultural interaction. And the cognitive linguistics approach both admits the linguistic relativism and tries to offer a solution to the disparity in cultural connotations of the poetic imagery. Nevertheless, with the duel between domestication and foreignizaiton pacified by the cultural interaction, more poetic images would be preserved in translating Chinese poems into English.
\end{abstract}

Keywords: Poetic images, Loss of gain, Image preservation

\section{Poetic images and the problem of cross-cultural understanding}

In literary criticism, the imagery is at least used in three senses. In the broadest sense, the images need not be mental "pictures", but may appeal to other senses other than sight. They might include auditory, tactile, thermal, olfactory, gustatory, or kinesthetic, as well as visual qualities. More narrowly, imagery is used to signify only descriptions of visual objects and scenes. And in the opinion of New Criticism, imagery signifies figurative language, especially the vehicles of metaphors and similes.

As they concern the poetry, images are usually defined as the concrete representation expressive or evocative of something else. The imagery that conglomerates the set of images in a specific poem enlivens it through reviving the imagination of its readers and bringing them into the perceptible world of sights, sounds, smells and sensations. Thus images are considered by some critics of poetry as its life and soul. Interestingly, the Chinese equivalent for the English word "image" is composed of two characters, that is, $Y i$ (connotation) and Xiang (physical image). Then the image becomes both associative and emotionally appealing that amalgamates the physical image with its spiritual connotations. The Chinese equivalent that emphasizes both the concrete and abstract aspects of a concept suggests that the image does not exist for its own sake but to invoke a sensation and the imagery is usually culturally-bound. This also conforms to Pound's definition of an image as an intellectual and emotional complex in an instant of time, as a vortex or cluster of fused ideas endowed with energy (Raffe, 1984). Then the imagery may be anything that a poet includes in his poem to connote a sense beyond the surface interpretation.

The classical Chinese poetry abounds particularly in images. And in choosing these poetic images, the classical Chinese poets have their preference for certain animals, plants and natural objects, either to cater to the interests of Chinese 
readers or their own idiosyncrasies. Just as the rules and forms of classical Chinese poetry are rigid, so is the choice of images to appear in these poems, for the beauty just lies in the images contained in them to describe and depict scenes and show the poets' feelings. To appreciate the magical images the readers are expected to get the same somatic experiences as the poets when they composed them, to appreciate the sceneries depicted and understand the emotions expressed by the poets and have a taste of the imagination. Since "languages differ in the way they perceive and partition the reality (Hatim \& Mason, 2001:104)", the Chinese poets' preference for certain images in their poetry certainly reflect a different perspective of poetic images than the English poets might hold about them. Moreover, due to the geographical barriers that used to separate China from the rest world, it is not easy for people from other nations to understand these images in the same manner, especially when they are reading the Chinese poems in the translation versions.

For example, the four flowers, chrysanthemum $(\mathrm{Ju})$, orchid (Lan), plum (Mei) and bamboo (Zhu), cherished by classical Chinese poets seldom appear in the English poems (even when they appear in a poem, they never constitute important images). Nevertheless in China, they are the so-called "four gentlemen (Jun $\mathrm{Zi}$ in Chinese, which is itself a different concept from gentleman to some extent) in flowers" of great significance to the poet and their poems. In the conceptualization of a native English speaker where the "bamboo" may even not be regarded as a flower, how can it be associated with a gentleman? Then the rendition of these flowers poses a problem to the translator of Chinese poetry. Should he or she keep the original image in the translated version or substitute it with another image? The problem is further complicated when the alias for a flower is used. For example, when the chrysanthemum is called "Huang Hua", should it be translated back into "chrysanthemum" or into "yellow flower" directly?

\section{Loss or Gain}

According to Croft \& Cruse (2006:19), "the profile-frame/domain distinction is particularly useful in understanding the nature of semantic differences between words and their apparent translation equivalents in different languages. The profile-frame/domain distinction may shed light on some aspects of why translation is difficult and often unsuccessful." By citing the example of the Javanese word rasa, Croft \& Cruse (2006:19) further points out, "basically, understanding the meaning of rasa presupposes large portions of Javanese culture and worldview." Their viewpoint towards the understanding of a word borders on that proposed by a functional linguist, who emphasizes the role of context in nailing down the meaning of a particular word. Then comes the problem of translating the classical Chinese poetic images. When these images are plucked out of the Chinese cultural context and translated into English, how can they be correctly understood by the target language readers?

Due to the cultural differences, it is usually difficult to find the equivalent in English for an image contained in the Chinese poetry. There has long been serious concern in the translation circles over the misrepresentation of the Chinese poetic images in the English language. And the concern over the loss of poetry in the process of translation has led quite a few literary scholars and translation theorists to propose the impossible task of poetic translation. Back to the understanding and translation of the "four gentlemen in flowers", they surely pose great challenges to the translator of classical Chinese poetry. Take the poem "A Song of Changgan" composed by Li Po as an example. The famous quotes from this poem, "Lang Qi Zhu Ma La, Rao Chuang Nong Qing Mei" include two Chinese poetic images. When they are translated into English as "When you, my lover, on a bamboo horse, /Came trotting in circles and throwing green plums" without any notes, the English reader most probably will be puzzled by these images. The problem does not lie in that they do not know the two images, but that they do not understand their cultural implications to the Chinese poets and the Chinese readers of poems. This concern often leads to the pessimistic viewpoint that culturally-bound poetic images are untranslatable. Moreover, the poetic translation with notes is often rebuked by the idealists as non-poetry, for they deem that "unlike other forms of literary translation, the translation of poetry must stand on its own as a poetic text, to a large extent unsupported by glosses or commentary, whether they take the form of footnotes or are embodies in the text (Connolly, 2004: 171)."

Despite the above-mentioned concerns over the loss, the practice of poetic translation has long in existence from English into Chinese and the back way around. According to Qian Zhongshu's research, the first translation of a Western poem, A Psalm of Life by Henry Wadsworth Longfellow can be traced back to the 1860s. Since then, the translated Western poetry has constituted an important part of the Chinese publications of poems. On the other hand, the translation of Chinese poetry, especially the Tang poetry, into English has also aroused interest among the Western scholars. Among the English scholars are such famous scholars as Arthur Waley, Burton Watson, Stephen Owen. What is more important is that during the course of translation, they have noted the significance of translating the poetic images from Chinese into English. According to Arthur Waley (Xia \& Wang, 2000: 270), imagery is the soul of poetry, so he proposes the principle of not adding to the imagery or deleting any original images.

In view of hermeneutics, even the comprehension of the original text varies to different readers in different epochs. With the lapse of time, different interpretations have been offered for the same poetic image from the Chinese poetry. At the very outset, the translation of Chinese poems is open for different interpretations. It is useless to lament over the 
possible "mistakes" existing in the poetic translation practice, but more important to take a general view of it to see what those practitioners have done to bridge the cultural gaps. To cite George Steiner (2001:311), "often, in the records of translation, a fortunate misreading is the source of new life. The precisions to be aimed at are of an intense but unsystematic kind. Like mutations in the improvement of species, major acts of translation seem to have a chance necessity. The logic comes after the fact. What we are dealing with is not a science, but an exact art."

To follow the hermeneutic approach that emphasizes the translation practice, examples are cited here to illustrate the image translation. First, take a look at the image of "chrysanthemum" in the classical Chinese poems and how different translators have dealt with the image. According to Gao \& Gao (2003: 85), as an important image in traditional Chinese culture, chrysanthemum represents a noble and unadulterated mind. Due to the repeated allusions to this flower in different poems, the chrysanthemum has been closely associated with lonely and proud hermits as well as literary men. Furthermore, through the device of synecdoche, the flower is frequently referred to as a "yellow flower" or "yellow" itself and there forms an intertextuality between "Huang Hua (yellow flower)" and "Ju Hua (chrysanthemum)" in the Chinese context. Gao \& Gao in their paper explore the different English versions of "Huang Hua" in Li Qingzhao's stanza of a song lyric, “Man Di Huang Hua Dui Ji. Qiao Cui Shun, Ru Jin Shei Kan Zhai?”. Li Qingzhao is one of the most talented Chinese poetess in the Song Dynasty and even the whole history of Chinese literature. After commenting respectively on Xu Yuanzhong's "yellow flowers", Kenneth Rexroth's gold chrysanthemums" and John A. Turner's chrysanthemums, they conclude that Xu has not conveyed the exact meaning of the allusion in Li's poem by translating it word for word into English as "yellow flower. They appreciate Rexroth's version of "gold chrysanthemums" by saying that it goes back etymologically to the Greek source of this English word.

However, "gold chrysanthemums" is a little repetitive for the meaning of "gold" has already been inherent in the word "chrysanthemum." Back to the question of intertextuality, the association in the Chinese language of the color yellow with the flower of chrysanthemum is lost when the image is replaced by the word "chrysanthemum" in English. The loss will never be regained if the readers do not go to the original Chinese version of the poem. That explains why translators often resort to interpretation to give the target language readers some general knowledge about the alien culture that forms the backdrop of the literary work they are going to read. Hawks \& Minford has even written a whole book to give explanations to how they have translated "The Story of the Stone" from Chinese into English. And that is where the readers of this ancient Chinese novel might gain their knowledge about the Chinese history and culture. The translation loss more often than not inspires the readers to explore the original.

\section{Possibility of image preservation}

The possibility of preserving the poetic images in translation lies in the similarity that exists to some extent in poetic images between English and Chinese. Without considering the "misreading" as concerns many scholars, there are certainly equivalents (in surface meaning) in English for quite a lot of poetic images in Chinese. The direct transplanting of such images from Chinese into English sometimes represents an effort to keep the original flavor of a Chinese poem if it is not a result of the laziness of the translator. However, this effort might be frustrated by the resistance from the target language readers if they can not associate this transplanted image with something they have been familiar with. The comparative literature and the studies can do a good job here.

For example, both the Chinese and the English peots have used the image of "crow" in their peotry. Generally in the Christian culture crow means nothing good though historically some European nations appreciate its sagacity. So in the English versions of the Chinese poem "Night Mooring at Fengqiao Village" by Zhang Ji, the image of "crow" is retained and the line "Yue Luo Wu Di Shuang Man Tian" is respectively translated as "The moon is sinking; /A crow croaks a dreaming" by Sun Dayu, and "The moon goes down and crows caw in the frosty sky" by Xu Yuanzhong. In English literature, Edgar Allan Poe employs the image of "raven", a similar bird to crow, in a famous poem for the melancholy tone and the symbolism of ravens as birds of ill-omen. And Ted Hughes refers to the bastard crow in his poem titled "King of Carrion" (Bebergal, 2006). Thus a rough equivalence is obtained between the Chinese image of "crow" and the image of "crow" or "raven" in English.

However, the case for preserving a poetic image is rather a complicated process. The Chinese poetic image, say, "oriole" is misrepresented by some in English as "nightingale" for the bird nightingale is a frequently adopted allusion in the western poetry. John Keats is greatly saddened on hearing the plaintive song of nightingale that inspires him to compose "Ode to a Nightingale". Though the image is so popular with the English readers, the European songbird with reddish-brown plumage noted for the melodious song of the male at night during the breeding season certainly is not an oriole popular with the Chinese poets and readers. Then the image must be borrowed into English in the process of translating a Chinese poem containing such an image. Most translators do so. The lines "Da Qi Huang Ying Er, Mo Jiao Zhi Shang Di" from a classical Chinese poem "Complaint in Spring" by Jin Changxu are respectively translated into English as "Oh, drive the golden orioles, from off our garden tree" by Fletcher and "Drive orioles off the tree, /For their songs awake me" by Xu Yuanzhong. And Giles has translated Du Fu's poetic line "Liang Ge Huang Li Ming Cui Liu" into "Two orioles sit in the green willows singing." Though orioles are not so frequently included in English poetry as 
an image, there is an exception offered by Emily Dickinson in her "To Hear an Oriole Sing", "To hear an oriole sing /May be a common thing, /Or only a divine." In this poem, oriole is depicted as a cheerful bird that expresses a happy emotion. Thus comparative studies in literature often reveal some similarities across cultures unexpected by many. And this is surely important to the translation theory and practice.

Of course the matching in imagery between Chinese and English literature is not a universal phenomenon. For example, translation of the natural image "Xi Feng" in classical Chinese poems always pose a great challenge to a translator. When the poetic lines "Mo Dao Bu Xiao Hun, Lian Juan Xi Feng, Ren Bi Huang Hua Shou" by Li Qingzhao tuned in the song lyric name "Tipsy in the Flower's Shade (Zui Hua Yin)" are respectively translated into English as "Should western wind uproll the curtain of my bower, / T T would show a thinner face than yellow flower" by Xu Yuangzhong and "The west wind rolled up curtains in shadow, /To show a leaner face than flowers yellow" by Tang Kuizhang, the west wind does not guarantee any understanding of the chilliness of the wind on the part of the native English readers, for they are so familiar with the poem "Ode to the West Wind" by Percy Bysshe Shelley where the west wind symbolizes hopes and force. In this particular instance an explication about the difference between the English and the Chinese culture is required. That is to say, a hermeneutic approach is involved to allow the alternity to exist in the target language and culture. However, some scholars argue for a misreading. Fan Jinglan (2008: 53-57), concerned with comparative literature, studies the imagist poets' misreading of the classical Chinese poetry. She concludes that "misreading" can be said to be a normal phenomenon occurring in the interaction of two different cultures. Both intentionally and unintentionally, the "misreading" offers a more valuable space for literary development and recreation. Therefore, in Fan's viewpoint, the translation of Xi Feng into the west wind is an unavoidable misreading.

Aimed at a systematic solution of the cultural disparity in translation, Wang Ying (2004: 584), starting from the embodied philosophy and cognitive linguistics, offers a cognitive linguistics model of translation and proposes the realization of "rational explication" and "harmonious translation". He also emphasizes the interaction between translator and text, between translator and real world, between translator and reader. Like Croft \& Cruse (2006), he also admits the cognitive binding on the understanding and translation of a text. Then the cognitive disparity between different languages and the barriers that ensue between the original poet and the translator must be dealt with meticulously.

\section{Conclusion}

The translation of images in classical Chinese poems into English is a strenuous job. As the result is concerned, the poetic images might be preserved, lost or replaced by others. Different approaches to the poetic images in translation put emphasis on different aspects of the same issue. The hermeneutic approach, with a view to preserving the alterity of an alien culture, would like the original image to be preserved as much as possible. The comparative literature approach is concerned with the misreading unavoidable in the translation process and cultural interaction. And the cognitive linguistics approach both admits the linguistic relativism and tries to offer a solution to the disparity in cultural connotations of the poetic imagery. Nevertheless, with the duel between domestication and foreignizaiton pacified by the cultural interaction, more poetic images would be preserved in translating Chinese poems into English.

\section{References}

Bebergal, Peter. (2006). Peter Bebergal reflects on crows, hawks and the loss of his mother. [Online] Available: http://www.jewishquarterly.org/article.asp?articleid=195

Connolly, David. (2004). Poetry Translation. In Mona Baker. Routledge Encyclopedia of Translation Studies. Shanghai: Shanghai Foreign Language Education Press, p. 171

Croft, William \& D. Alan Cruse. (2006). Cognitive Linguistics. Beijing: Beijing University Press, p. 19

Fan, Jinlan. (2008). On the "misreading" of Imagists on the Classical Chinese Poetry. Journal of Southwest China Jiaotong University (Social Sciences Version), Vol. 9, No. 3

Gao, Lu \& Gao, Yuan. (2003). "Huang Hua"="yellow flowers"? —on the necessity of introducing the theory of intertextuality into translation studies in view of the C-E translation of a Chinese poem. Journal of Anhui University (Philosophy and Social Sciences Version), Vol. 27, No. 4

Hatim, Basil \& Ian Mason. (2001). Discourse and the Translator. Shanghai: Shanghai Foreign Language Education Press. p. 104

Raffe, L B. Ezra Pound. (1984). The Prime Minister of Poetry. Hamden: The Shoe String Press.

Steiner, George. (2001). After Babel: Aspects of Language. Shanghai: Shanghai Foreign Language Education Press, p. 311

Wang, Ying. (2007). Cognitive Linguistics. Shanghai: Shanghai Foreign Languages Education Press, p. 584

Xia, Kongda \& Wang, Xiaoping. (2000). Foreign Studies on the Chinese Literature in the $20^{\text {th }}$ Century. Tianjin: Tianjin

People's Press, p. 270 